\title{
Molecular Nanotechnology: A new avenue for Environment Treatment
}

\author{
Manju Singh ${ }^{1}$, Naveen .B.P $P^{2}$ \\ ${ }^{1}$ University of Genoa, Genoa, Italy \\ ${ }^{2}$ Department of Civil Engineering, Indian Institute of Science, Bangalore, India
}

\begin{abstract}
In the environment, nanotechnology can add significant improvement by providing their role in monitoring, pollution prevention and remediation methods. There are a number of promising areas of applications, such as contaminated water and air treatment, self-cleaning materials, energy applications, novel functionalized adsorbents for environmental, industrial applications and nanomaterials for sustainable energy production. Moreover, there are also naturally occurring nanomaterials, which can strongly affect the quality of the environment. In this paper, we will address some of the advances method and their role which are being applied to provide more sensitive detection techniques for air and water quality monitoring. New synthesis methods for effective metal oxide nanocatalysts will help reduce pollution because of transport and industrial sources. The photocatalytic properties of titanium dioxide nanoparticles are being exploited to manufacture "self cleaning" architectural coatings that are capable of degrade nitrogen oxides, volatile organic compounds (VOCs) and other pollutants into less toxic species.
\end{abstract}

Key words: Environment, Monitoring, Pollution, Remediation, Water treatment.

\section{Introduction}

Nanosciences and nanotechnologies have been recognized as one of the most progressive and fast growing frontiers in the world of $21^{\text {st }}$ century. By adding, Nano-materials, due to their unique properties such as high specific surface area, high surface energy etc. are mainly used in modern industry and life sciences areas especially in the field of environmental protection. Human race accompanied by other species on earth is victimized by soil, water and air pollutions, threatened by hazardous and nuclear wastes by current state-of-art technologies. Global warming, ozone depletion, acid rain has become household daily vocabularies. Environmental sustainability based on technology-to-date is negative. In all industrial fronts, such as nanobiotechnology, nanomaterial, nanoelectronics, nanoenergy, and etc., offer radical tools for human society for the first time to be on the upper hand in the struggle toward sustainable economic growth by emerging molecular nanotechnology. Furthermore, it will have more capability for human civilization not only to remediate environmental problems accumulated since industrial revolution of $18^{\text {th }}$ century, but also to produce indefinite energy and material with ultra-green processes. In the process of degradation of organic pollutants in water, the conversion of the toxic ions from more values to less ones which will have small toxicity or even no toxicity at all, and the purification of the vehicle exhausts by using nanomaterials. This technology is allocated into two main research areas, the first one is the traditional solid-phase nano-materials applications which is based on the study of solid-liquid interface reaction and process to target the aim of removing pollutants in the soil and water and the other is gaseous nano-materials. By using the solid particles to load gaseous nanoparticles as $\mathrm{O}_{2}, \mathrm{O}_{3}$ to improve the stability as well as the availability of gas transportation, therefore provide a novel technology for the improvement of lake sediment and the deep treatment drinking water. This paper suggests the causes of sustainability problems and diagnoses the defects of current industrial manufacturing processes by enlighten the role of molecular nanotechnology. This paper also induces and analyzes the vision of further competences that human may gain from the development of nanotechnology that have the likely to ascertain environmental sustainability, renovate global environment whereas we still enjoy the plenty of material and energy. Nanotechnology is an essential component in the development of improved systems for monitoring and cleanup including all the three phases of environment figure 1. Detection and measurement of pollution is also a necessary step towards initiating remedial action.

\section{Contaminated Environment and Molecular Nanotechnology (MNT) Methods}

We have stored a serious environmental obligation $(1,2)$ that results in numerous potentially harmful problems from $18^{\text {th }}$ century industrial revolution to $20^{\text {th }}$ century information age. In which, the most tarnished are acid rain, ozone layer depletion, global warming, contaminated water and soil, and large piles of nuclear waste. We can only deal with the most threatening by transferring it to less threatening, "out of sight, out of mind" is the general adopted philosophy because of economic and technological limitations. Now, first time in human history, MNT offers us the capability to pay off the stored debt and return nature to its original state. All 
human activities are inspired by survival. For that, human produce material, consume energy and products. The technology employed for production is a top-down bulk technology (TDBT) before molecular nanotechnology (MNT) whether it's of traditional or of high technology. Because of TDBT's inherent shortcomings, such as low efficiency, inaccurate, crude, and etc., energy and material are excessively wasted; pollutants and wastes are extensively generated throughout the processes. Additionally, human consumption also consumes enormous quantity of energy and material which generates intense amount of pollutants and wastes. All these threaten the sustainability of our environment. Product manufacturing, energy production, material production, and consumption can be concise as human survival activities. Because of technology-to-date (TTD) is original in contrast with the emerging MNT; all human activities are applying huge pressure on the environmental sustainability. The product based on MNT is different from nanoscale material, as it is based on the ability to build structures to complex, atomic specifications by means of mechanosynthesis. Richard Feynman's vision of miniature factories using nanomachines to build complex products, this advanced form of nanotechnology or molecular manufacturing. It would make use of positionally controlled mechanosyntheis directed or molecular machine system. MNT would contain combining physical principles established by chemistry, nanotechnologies, and the molecular machinery of life with the systems engineering principles started in modern macroscale factories, see figure 2

\subsection{Acid Rain and Smog}

Acid rain is one of the most important environmental problem of all, cannot be seen. It is well known that nitrogen oxides (NOx) and sulfur dioxide $\left(\mathrm{SO}_{2}\right)$ are the primary causes of acid rain and smog $(3,4)$. Power stations, factories and car all burn fuels and therefore they all produce polluting gases. Some of these gases (especially nitrogen oxides and sulphur dioxide) react with the tiny droplets of water in clouds to form sulfuric and nitric acids. The rain from these clouds then fall as very weak acid, which is why called as acid rain. Acid rain is the reasons of acidification of lakes and streams and to damage of forests and forest soils. Moreover, acid rain increases the decay of building materials, paints, wall coating, including old buildings, statues, and sculptures that are part of our civilization's heritage (5). The strength of the effects depends inversely on buffer capacity of the waters and soils involved. $\mathrm{SO}_{2}$ and $\mathrm{NOx}$ gases and their derivatives as sulfates, nitrates and ozone, add to reflectivity degradation and harm public health (4) in the atmosphere. Once MNT empowered solar energy become exclusive source of energy as a long-term energy umbilical cord that links with fossil fuel can finally be severed. In addition, nanomaterial which used to constructed future vehicles driven by nanoelectro mechanical system and powered by solar cell or hydrogen fuel cell will totally remove transportation related $\mathrm{SO}_{2}$ and $\mathrm{NOx}$ emission. So, the anthropogenic $\mathrm{SO}_{2}$ and $\mathrm{NOx}$ that attack our atmosphere since industrial revolution can be stopped; thus further acidification to the environment and threatening to human health by adding toxic gases can be relieved. Nanobots such as nano-desulfurizer can be sent up to the atmosphere to capture $\mathrm{SO}_{2}$ gas and reduce it to sulfur and precipitate to earth surface as dust. As, nano-sulfurprecipitator contains calcium or magnesium ion that can be sent up into sky to oxidize $\mathrm{SO}_{2}$ and formed $\mathrm{CaSO}_{4}$ or $\mathrm{MgSO}_{4}$ salts. More, nano-catalytic-converter, convert $\mathrm{NOx}$ into nitrogen and oxygen. Nanobots like nanoNOx-reducer can be sent up to capture NOx and transform it into ammonia and bring it down to the ground in case of agriculture technology still needs fertilizer. We can distribute a troop of nano-buffer to increase their buffer capacity in resisting acidity for ground level treatment, the acidified water bodies and soil. Either by capturing $\mathrm{H}^{+}$from the environment or giving off $\mathrm{OH}^{-}$to the environment, we can also deploy an army of nanoneutralizer to vigorously adjust $\mathrm{pH}$ in water or soil to their original condition.

\subsection{Global Warming}

A regular rise in the average temperature of the Earth's atmosphere and its oceans, a change that is believed to be permanently changing the Earth's climate is defined as a global warming. The excess overloading our atmosphere with greenhouse effect gas- $\mathrm{CO}_{2}$ comes from the fossil fuels we burn for energy (coal, natural gas, and oil) plus the loss of forests due to deforestation, especially in the tropics. 2012 was the hottest year ever recorded for the contiguous United States. Before MNT, about 300 billion tons of excess $\mathrm{CO}_{2}$ have been added to the atmosphere (6). Climatologist's project that hiking $\mathrm{CO}_{2}$ levels, by setting up solar energy, will partially melt the polar caps, raising sea levels and flooding coasts sometime in the middle of the next century (7). By applying MNT to energy production will support solar power to be generated at an affordable cost. This will not only eliminate fossil fuel power generation but also $\mathrm{CO}_{2}$ emission and thus relieve the fear of a flooding catastrophe. MNT will also facilitate us to develop nanomachines, nanobots as nano-photosynthesizer $(8,9,10)$ nano-carbon-fixer, nano-chlorophyll, etc. A nanobot's power source depends on its environment. The nanobot's efficiency and small size means it would have less control on the host person. A free-ranging nanobot could take energy from sound or radio waves beamed from outside. Independently driven nanobot would require allocating most of its bulk to energy storage in the form of chemicals or other systems. These nanobots not only can be operated to remove all the 300 billion tons of excess $\mathrm{CO}_{2}$ from atmosphere, but also can transform them into 
valuable materials by driving cheap solar energy. The carbon removed by nanobots can be used in synthesizing functional and structural materials. It can also be removed by other nanobots and further synthesized into sugar, starch, and cellulose to complement our demand for food, paper and etc. This cans for the time being relief the pressure which we apply on farmland and forest. We can place it back into the coal seams and oil fields from which it came, if there should have excess carbon continued.

\subsection{Ozone Layer Depletion}

CFCs (chlorofluorocarbons) and halons are most threating to the ozone layers (11). Winds proficiently mix the troposphere and equally dispense the gases. CFCs are very stable, and they do not dissolve in rain. After a period of numerous years, ODS molecules reach the stratosphere, about 10 kilometers above the Earth's surface. Strong UV light breaks apart the ODS molecule. CFCs, HCFCs, carbon tetrachloride, methyl chloroform, and other gases release chlorine atoms, and halons and methyl bromide release bromine atoms. Before it is removed from the stratosphere or becomes part of an inactive compound (12) each molecule of chlorine in CFC have a capacity of degrading more than 100,000 molecules of ozone. As per pervious studied, in 1991, 682 million kilograms of CFCs is consumed globally. In which maximum 32 percent for refrigerants see table 1. The Montreal Protocol is enrolled by world governments to protect the ozone layer $(15,16)$ as an international agreement because to see the destructive power of these ozone-depleting substances (ODS) (13, 14). The relative influence of the diverse ODS depends on the stability of the reservoir compound such as $\mathrm{HBr}$ and $\mathrm{BrONO}_{2}$ of bromine reservoirs are 10 to 100 times more effective than chlorine at destroying ozone (12). The non-reactivity of ODS is required in industry by allowing them to drift for years in the environment until they finally reach the stratosphere. Intense UV solar radiation splits halogens off of the ODS, and these unattached halogens that are able to catalytically convert ozone molecules into oxygen molecules (13) in the stratosphere. To remove from the stratosphere diverse ODS require diverse time which would be about 50 to over 200 years. It is encouraging to see that the growth-rate of ODP in the atmosphere is starting to drop, without MNT stepping in, the impact of ODS on stratospheric ozone will continue well into the 22nd century. MNT primarily will cautiously use water as solvent, and can 100\% recycle them (2) in the future. ODS refrigerants can be replaced at a higher cost even at this pre advance stage. MNT will help lesser that cost to negligible. Consequently, the growth rate of ODP in ODS reservoir will become zero. Drexler (2) proposed using sodium containing balloon type nanobots for the ODS remaining in the reservoir. The nanobots powered by nano-solar cells collect CFCs and separates out the chlorine in the stratosphere and by combining this with sodium makes sodium chloride. The balloon collapses and falls when the sodium is departed. A grain of salt and a biodegradable speck fall to Earth in the end. The stratospheric CFC can be removed rapidly. Nanobots containing other metals for example magnesium and calcium can also be planned to eliminate stratospheric CFC encompassing Drexler's thought. In the same tactics, among ODS, halogens expect chlorine such as bromine can be neutralized and to deploy an army of airborne solar energy powered metal containing nanobots into the stratospheric ODS reservoir. ODS impact can be reduced after the actual deployment date of the halogen neutralizing nanobots within 200 years of continuous efforts. ODS reservoir in the stratosphere can be resolved by solar powered airborne metal nanobots to reduce ozone depletion problem is a conceptual idea. This is represented in figure 3 .

\section{4 Soil and water contaminations and toxic wastes}

It is a big concern that living systems harms by toxic wastes which are collected in piles or in contaminated surface water, groundwater, or soil. It can be organic or inorganic contaminants. The inorganic contaminants contain toxic elements, for example mercury, lead, cadmium, aluminum, manganese, selenium, and arsenic and the organic contaminants, such as Dioxin, Polychlorinated Biphenyl (PCB) and etc. Worldwide existing "Love Canal" contaminated dumpsites can be easily clean up with the vision of MNT. The liquid and solid waste material will undergo a treatment to break the matter down into its chemical building blocks. Because of this method, it will be possible to recycle unbrokenly and recreate any type of material. Products for recycling will be arranged by microscopic nanobots that separate mixtures of materials into classified based on their size, shape, colour and on their physical and chemical properties. Specific nanobots for attacking specific contaminants at their weak points can be planned after knowing the chemical properties of the organic contaminants. An army of these nanobots can then be deployed into contaminated site; either it is in soil or water and to extract these contaminants harmless by rearranging their atoms. The nanobots can be designed in a way that no recovery needed after mission completed. They can be recovered and reprogrammed for other cleaning tasks after each task if it can be designed reprogrammable. By specially designed nanobots inorganic contaminants can also be collected. To identify these toxic elements and store them inside, trillions of them can be sent into contaminated waters bodies or soil. The nanobots are then recovered from the site, initiated to release toxicants, and then ready to perform other tasks. By nanobots the released concentrated metal ions can be restored into rocks in the mines or fused to stable form by nanomachine and returned to nature. 


\subsection{Nuclear Waste.}

The most significant high-level waste from a nuclear reactor is the used nuclear fuel left after it has spent. Although, MNT can only work with atoms and molecules, not with nuclei so that MNT cannot treat nuclear wastes and reduce their harm directly. However indirectly, MNT can offer us the means for a clean, permanent solution to the untreatable nuclear wastes left over from the nuclear age by reducing the cost of energy and equipment. By using specify nanobots nuclear wastes can be collected and concentrated. Products of MNT could help with forms possible conventional methods to dealing with nuclear waste, helping to store it in the most stable and reliable. We could seal them in self-sealing containers and driven by cheap nano-solar energy (6) by using nanomachines. These would be safer than any other passive cask or rock. The concentrated nuclear wastes can be transported to the moon and bury them in moon's dead, dry rock by nanobots, or to other planets that still radioactive, or even shoot them directly into the sun by using reliable and cheap spacecraft with advancing in MNT. Underground nano-atom smasher driven by cheap solar cells can also be planned to treat nuclear wastes. This is a reverse process of nuclear engineering. The nanomachine will smash radioactive target and harvest for nonradioactive substance at the place of smashing nonradioactive target and harvesting for radioactive substance. The smashing and harvesting process will remain stable is attained. Figure 4 illustrates a few ways for resolving nuclear waste piles that collected in the environment and TDBT is at loss on dealing with them.

Additionally, recently scientists have discovered that radioactive materials in water can cluster onto flakes of graphene oxide (GO). Researchers predicted that $\mathrm{GO}$ could sense and identify extremely low levels single molecules of various substances based on theoretical models and calculations. Using the latest advances in supercomputing, they modeled several different variations of GO to figure out which one would be the most sensitive and selective in detecting uranium and plutonium in nuclear waste water. They concluded that attaching something called a carbonyl functional group to GO would serve as an effective nanosensor for these radioactive materials.

\section{What are the potential risks of using Molecular Nanotechnology in remediation applications?}

A product must be tested for safety before it is applied to market. There is no different for nanoproducts and processes, this is the rule for all products - drugs, chemicals, materials, etc. By properly and thoroughly tested of a product the potential risks will have been known. Some of the problems with nanoparticles have been defined here.

$>$ Long-term act not as good as promised (remediation goals not being met)

Incorrect transport and reaction behavior predicted (consistency not correctly understood).

> Perception as a contaminant if nanomaterials are used in situ (parallel to GM plants seen a contaminants rather than beneficial in crops.

$>$ Unknown side effects arising from moving to field applications too soon.

If products are more toxic than the original pollutants, it might be one of the potential dangers of environmental remediation. Other risks are signified by access of toxic by-products into the food chain, soil degradation and plant pathology. Abandoned contaminated industrial and military sites are old and the most urgent problems for remediation. Some of the contaminants of particular interest are trichloroethene (TCE), trinitrotoluene (TNT), Cr (VI), As (III). The application of nanoparticles for soil remediation is presently under examination. Zerovalent iron and iron oxides, catalysed by noble metals such as Pd are the material which is under consideration. Permeable barriers with iron filings are already used to remediate groundwater. Further studies of iron nanoparticle are needed of agglomeration/aggregation although it represents a more cost effective solution as they have a much higher reactivity per unit mass. There are still serious issues regarding the effect of nanoparticle release on soil ecosystems. This is big research challenge as there are limited data available. Among fundamental necessities are physico-chemical equilibrium and kinetic data, information on effects on living organisms and development of modeling methods on a case by case basis. Other techniques for nanoparticle remediation by using of micro-organisms and phytoremediation methods is need to be included. The complexity of systems involved means that there are many unknowns. It is therefore necessary to establish a system of defining acceptable and unacceptable risks.

\section{Additional research is needed to exploit the full potential of nanotechnology for remediation}

To apply various nanotechniques at nanoscale level to discover the nature's secret to understand the fundamental physical and chemical processes at work in nature. To improve the quality of air, water, and soil, advanced material developed by implementing those secrets. The significance of membrane technology in addition to the nanoparticles needs to be focused on. Nanotechnologies in Permeable Reactive Barriers to treat groundwater and using in situ release of reactive materials to support natural processes should have theoretical and bench modelling studies. For better understanding of nanomaterials: as sorbents in the environment, 
oxidation and reduction properties, to help monitor the environment, to deliver nutrients and other substances to help remediation processes must be combined. Basic research and investigations that will reveal the secrets of nature would be useful to improve the natural ability of soil, sediments and dust (natural nanoparticles) to eliminate contaminants. With the help of promoting fundamental research, new materials will apply the tricks of nature to make more proficient and sustainable filters for immobilizing contamination and eventually "mining" it from selective appropriation approaches. To better understand of membrane fouling can be developed by using Nano filtration in hybrid configurations, modelling and simulation studies. The feasibly toxic effects of nanoparticle release on ecosystems, including aquatic and soil research is certainly needed for better understanding. Further development in $\mathrm{TiO}_{2}$ photocatalysis for the control of indoor pollution and process water streams is needed. Proof-of-Concept and pilot trials are needed of $\mathrm{TiO}_{2}$ photocatalysis for urban air quality improvement. In permeable reactive barriers to treat groundwater, bench and pilot scale testing methods and better fundamental understanding of transport, reaction and fate in situ are needed for nanotechnology scientist. Nanotechnologies using in situ release of reactive materials to support natural procedures to attain particular results need bench-scale testing methods and better reaction and transport understanding.

More advance research is needed that control the treatment to remove highly toxic products. In order to know the potential risks of using nanoparticles for the purification of water and air can be detect by further research. Nano-enhanced membranes combine nanotechnology with membrane technology to improve membrane properties and thus increase their performance (17) such as there are several nanoparticles that can be functionalized in $\mathrm{TiO}_{2}$ to enhance its better filtrations process see table 2 .

\section{Conclusion}

MNT becomes an influential and achievable tool in our lifetime. We require a real sustainable environment to survive with a quality life. In order to restore the environment to its original perfect state and establish sustainability MNT has unlimited potential, we should focus on the unprecedented security and environmental benefits of MNT, as well as the potential problems. Yet, the development of MNT now is irregular; a greatly designed at additional economic growth and less is at environmental sustainability. MNT is vital for sooner understanding of MNT's benefits by system integration approaches for this need to educate MNT researchers about the potential benefits and risks of the technology, and introduce ethical guidelines into professional associations. Remarkably, the "moral repugnance" associated with biological weapons has greatly reduced their development and use, despite the fact that they are relatively easy to make and deploy. At the last, further research and development to environmental remediation is required for real environmental sustainability.

\section{References}

[1] K. E. Drexler, Engine of Creation, Healing And Protecting The Earth, Anchor Press, Doubleday, New York, 1986 http://www.foresight.org/EOC/section02of05

[2] K. E. Drexler, C. Peterson and G. Pergamit, Unbounding the Future: the Nanotechnology Revolution, Restoring the Environment, William Morrow and Company, New York, 1991 http://www.foresight.org/UTF/Unbound_LBW/chapt_9.html.

[3] National Science and Technology Council, Committee on Environment and Natural Resources, National Acid Precipitation Assessment Program Biennial Report to Congress: An Integrated Assessment; http://www.nnic.noaa.gov/CENR/NAPAP?NAPAP 96.htm

[4] United States Environmental Protection Agency (EPA), Environmental Effects of Acid Rain; http://www.epa.gov/acidrain/effects/envben.html

[5] United States Geological Survey, USGS Tracks Acid Rain; http://bqs.usgs.gov/acidrain/arfs.html

[6] D. Mulhall, Statement on NanoEcology, Foresight Institute, Palo Alto, California, 2002 http://www.foresight.org/nanoecology/Mulhall.html

[7] T.E.Graedel and P.J.Crutzen, Atmospheric Change: an Earth System Perspective, Freeman, New York 2nd ed, 1993

[8] Shapiro and et. al., Dependence of the luminescence energy in InGaN quantum-well structures on applied biaxial strain, Phys. Rev. B. 62, 2000, R16318

[9] Kazuhiro Sayama, Kazuaki, Mukasa, Ryu Abe, Yoshimoto Abe, and Hironori Arakawa, Photocatalytic Water Splitting System into $\mathrm{H} 2$ and $\mathrm{O} 2$ under Visible Light Irradiation Mimicking a Z-Scheme Mechanism in Photosynthesis, Solar Light Energy Conversion Team, Photoreaction Control Research Center, Advanced Industrial Science and Technology (AIST), Japan, PCPM2002, Poster Presentation http://www.aist.go.jp/aist e/new research/20011206 1/20011206 1.html

[10] K. H. Taylor, Artificial Photosynthesis, Basic Research in Biomimetic Applications: Multilayer Organic Photovoltaic Cell, CSIRO, Australia, 2002 http://www.tip.csiro.au/IMP/NanoScience/ArtificialPhotosynthesis/photovoltaicCell1.htm

[11] M. McFarland and J.Kaye Chlorofluorocarbons and ozone, Photochemistry and Photobiology, 55, 1992, 911-29.

[12] Washington University in St. Louis, Effects of Ozone Pollution http://www.nslc.wustl.edu/Outreach/currentSite/Ozone/troposphere/health.html.

[13]. J. C.Farman, B. G. Gardiner, and J. D. Shanklin, Large losses of total ozone in Antarctica reveal seasonal ClOx/NOx interaction, Nature, 315,1985, 207-10.

[14] J.G. Anderson, W.H. Brune, and M.H. Proffitt. Ozone destruction by chlorine radicals within the Antarctic vortex: The spatial and temporal evolution of ClO-O3 anticorrelation based on in situ ER-2 data. Journal of Geophysical Research 94 (D), 11, 1989, 46579

[15] Office of Air and Radiation (OAR), EPA, Proposed Rule for Reducing Regional Transport of Ground Level Ozone (Smog): Federal Register Notice; http://www.crest.org/clients/can/old/otagfr.htm

[16] The Montreal Protocol on Substances that deplete the Ozone Layer, United Nations Environment Programme, The Ozone Secretariat, 1987, http://www.unep.org/ozone/montreal.shtml 
[17] J Kim and B. V. der Bruggen, The use of nanoparticles in polymeric and ceramic membrane structures: Review of manufactureing procedures and performance improvement for water treatment, Environmental Pollution ,158, 2010, 2335-49

Table and figure of paper

Molecular Nanotechnology: A new avenue for environment treatment

\begin{tabular}{|l|l|}
\hline Sources & Percentage \\
\hline Refrigerants & 32 \\
\hline Blowing agents & 28 \\
\hline Cleaning agents & 20 \\
\hline Propellants. & 18 \\
\hline Other & 2 \\
\hline
\end{tabular}

Table 1: A studied of CFCs (chlorofluorocarbons) consumed globally during 1991 by different sources.

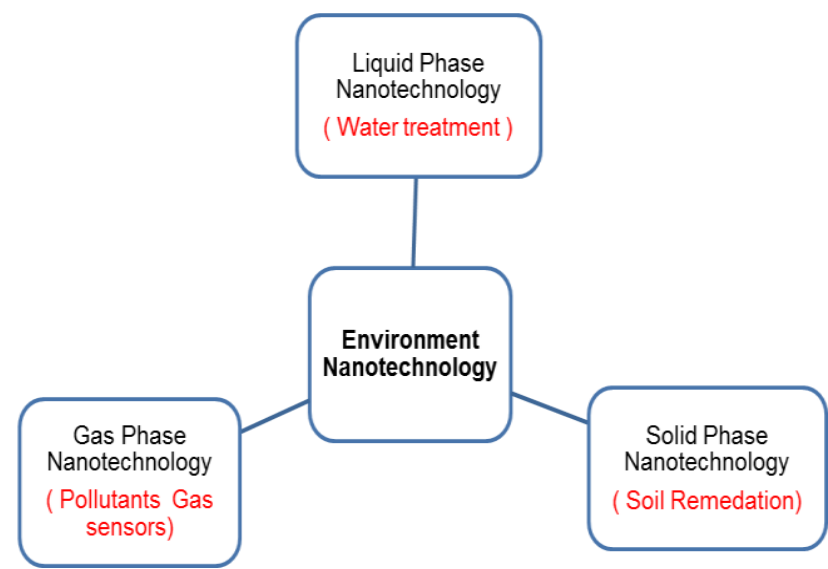

Figure 1: Application of Nanotechnology for Environment in all the three phases

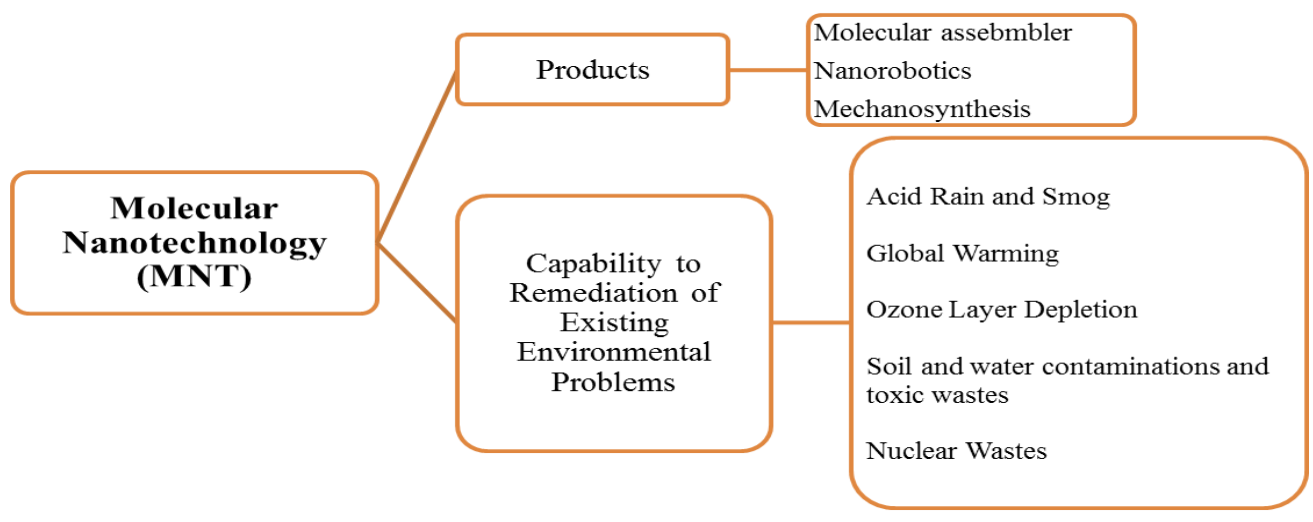

Figure 2: Products of Molecular Nanotechnology (MNT) and its capability to Remediation of Existing Environmental problems 


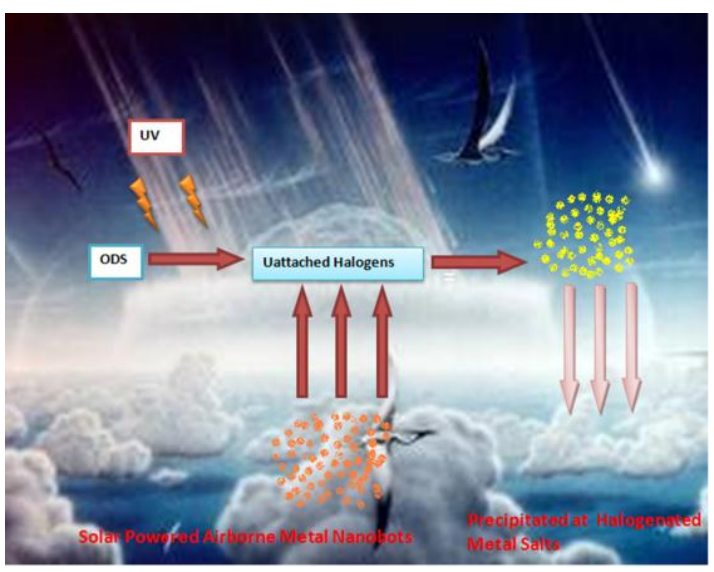

Figure 3: ODS (ozone-depleting substances) reservoir in the stratosphere by solar powered airborne metal nanobots to reduce ozone depletion.

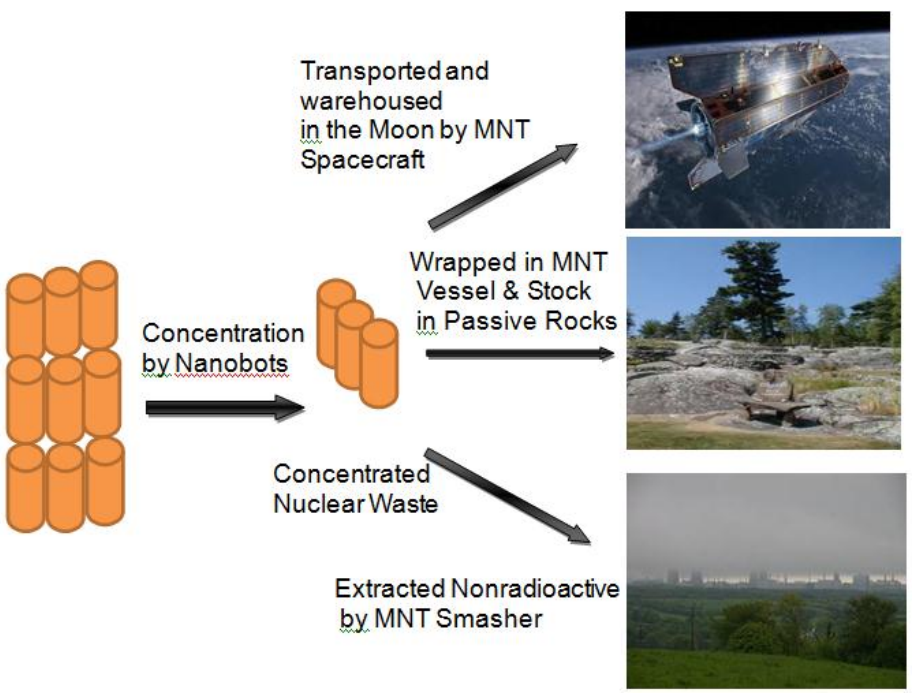

Figure 4: A way of MNT (molecular nanotechnology) to resolve collected piles of nuclear waste in the environment

\begin{tabular}{|l|l|l|}
\hline Type of Nanoparticle & Intended membrane improvement & Compatible membrane types \\
\hline $\mathrm{TiO} 2$ & Increased flux, antifouling & Ceramic, Metallic \\
\hline $\mathrm{Ag}$ & Antimicrobial & all \\
\hline $\mathrm{CNT}$ & Increased flux & N/A \\
\hline $\mathrm{Al} 2 \mathrm{O} 3$, Silica, Zirconia & Increased flux & all \\
\hline
\end{tabular}

Table 2 : Nanoparticle used in membrane 